\title{
UAV Positioning Search and Rescue System
}

\author{
Huaqing Liu ${ }^{a}$, Zhou Yan ${ }^{\text {b }}$, Jing Lei, Junfeng Fu, Hang Dong, and Xiao Liu \\ School of Engineering Training Center, Shenyang Aerospace University, Shenyang 110136, China.
}

Keywords: UAV, search and rescue positioning, video transmission, air-drop.

\begin{abstract}
The paper reviews the development of rescue drones. Combining with the mature research results of the market, the paper explores the principles of application and the realization methods of fixed point searching, real-time image transmission and emergency air-drop with UAV. A feasible UAV search and rescue system is designed.
\end{abstract}

\section{Introduction}

The drone is an unmanned aerial vehicle. In 1917, Britain took the lead in developing the world's first drone. After World War II, drones developed rapidly in areas such as reconnaissance and combat. Civil drones are more widely used, and have played an important role in aerial photography, agricultural flight defense, and electric power inspections.

\section{Advantages of Drones for Water Locate and Rescue.}

At present, the situation of people falling in large ports, river banks and other places is not uncommon. Due to the unknownness and uncertainty of the incident area, the difficulty of search and rescue is greatly increased. The traditional carpet-type search and rescue method is influenced by the environment and people's subjective factors. In complex situations, it is difficult for rescue forces to find the people in the water in time, but the drone rescue has the following advantages:

\subsection{High speed.}

The drone starts up quickly and can be on standby for a long time. Taking the electric drone as an example, the system can be dispatched after the power-on self-test is completed, which takes less than 5 minutes and is not interfered by human factors. The drone is light and has a good aerodynamic shape. The low-altitude cruising speed can reach more than 100 kilometers per hour, and it can quickly reach the location of the incident to carry out the search.

\subsection{Low cost and easy maintenance.}

Compared with manned aircraft, drones save a lot of equipment and have lower production and maintenance costs. Take the 40-meter patrol boat of Tianjin Maritime Safety Administration as an example, the fuel consumption is 0.25 tons per hour and the fuel cost is 2050 yuan. The fuel-free drone's fuel cost per hour does not exceed 200 yuan, and the patrol efficiency is several times that of the patrol boat. Secondly, the cost of the patrol boat is high. Take the 60-meter B-type patrol boat of Hainan Maritime Safety Administration as an example, each of which is 56.06 million yuan, and the single-machine price of the large-scale aerial survey drone that is used more is only 5,999 yuan.

\subsection{Mature technology and easy operation.}

The drone technology is very mature and can be executed with different tasks through reasonable design. Electronic equipment such as airborne photoelectric pods and atmospheric data sensors can be retrofitted on drones. The operation of the drone is simple, and the general technician can operate after short training. 


\section{The Application Technology of UAV Emergency Positioning Search and Rescue}

\subsection{Responding to take off.}

When the drone is on standby, it is in power-on, the image transmission system is turned on, and the positioning information is updated. The drone deployment site should have a flat runway of appropriate length and a simple hangar that can protect against wind and rain. The runway should be level so that the flight control device can self-calibrate. After receiving the distress rescue signal from the location beacon carried by the distress, the drone immediately takes off according to a predetermined procedure or under manual radio operation, and flies to the beacon signal location. Generally speaking, the $2.4 \mathrm{Ghz}$ radio multi-channel remote control commonly used in aviation models can be used for manual manipulation and flight control assisted operation. The A3 flight control built-in program has the functions of adjusting the rudder surface of the ailerons, elevators, rudders, flaps, etc., and balancing the center of gravity of the aircraft, limiting the pitch angle and the roll angle, etc., basically meeting the normal flight requirements.

\subsection{Search targeting.}

The positioning search and rescue system is mainly used in the field of water search and rescue, and it is necessary to install a radio positioning beacon in the lifesaving equipment. When the person falls into the water, the beacon will trigger an alarm when it encounters water, and automatically send a rescue signal with positioning information to the nearby maritime department headquarters or the shipborne information center. After receiving the warning signal, the search and rescue command center can select the drone to take off or manually take off according to the plan, go to the beacon to investigate, and coordinate with the surrounding rescue personnel to locate the search and rescue device to share the location information. When the drone approaches the beacon $10 \mathrm{~km}$ away, the onboard positioning signal receiver is activated, and the drone immediately approaches the beacon to accurately search for the target. The beacon standby time is not less than 72 hours, and the volume should be small enough to meet the reliability requirements.

\subsection{Long distance image transmission.}

After the drone is accurately positioned to the target, it starts to use the equipped long-distance image transmission device to perform image detection, and transmits the real-time video back to the command ground station through the transmission device. The rescue force can judge the state of the situation in time through the on-site image, determine the life status of the drowning personnel, and select an appropriate rescue method according to the site environment. At present, the long-distance video transmission technology is becoming more and more mature, and the anti-interference ability of the airborne transmission equipment is gradually strengthened. Take the Dabai aerial survey drone as an example. The orthophoto sensor is a 50.6 megapixel Canon 5DS full-frame camera. The single-pass time $10 \mathrm{~cm}$ resolution data is $30-60 \mathrm{~km} 2$; the tilt photography sensor (5S) is a 20-megapixel single camera, total 1 Billion-pixel camera, weighing less than $2500 \mathrm{~g}$, minimum exposure interval 1s; one frame to complete $8 \mathrm{~cm}$ resolution tilt photography data 3-6km2, with high-definition photography capabilities.

\subsection{Place items and equipment.}

Research on the casualties of drowning people shows that from the time when the drowning person is found to the time when the rescue force arrives at the scene, the probability of the faller encountering various complex environmental factors increases with time. Those who fall into the water must not only face environmental damage such as waves and floating objects, but also endure the deterioration of their own conditions. In addition, due to the influence of surface clutter, traditional search and rescue equipment finds that it is more difficult for those who fall into the water, and those who are in need of water are in urgent need of tools to keep in touch with the outside world. Therefore, the drone can be equipped with a simple delivery system. After the drowning person is found, the necessary rescue items such as precise positioning equipment, communication equipment, 
food replenishment and the like can be fixed.

\section{The Structural Design of the Unmanned Airborne Positioning Search and Rescue System}

\subsection{Unmanned airborne.}

The UAV of this project is based on the electric aviation model. The main materials are aviation laminate and balsa wood. The fuselage is $1.2 \mathrm{~m}$ long, the wingspan is $1.8 \mathrm{~m}$, the empty machine weighs $1.2 \mathrm{~kg}$, the payload is $500 \mathrm{~g}$, and the thrust-to-weight ratio is about 1.2 . The fuselage has a large capacity and has good conversion potential. The wing is a CLARK-Y subsonic airfoil with good low-altitude high-speed flight capability.

According to the test results, when the drone is equipped with A3 flight control, after the flight control power is turned on, the flight control self-calibration response time is about $3 \mathrm{~s}$, and the horizontal calibration is realized. The power unit is motor power. After the power is connected, the electronic governor immediately sets the throttle amount. The response time is about 5s. After the ESC mode responds, the motor is powered. Each rudder also completes the zeroing preparation during this period, causing the aircraft to take off.

\subsection{Image transmission device.}

In this project test, for cost considerations, the FPV Swift camera was used, with AOMWAY wireless image transmission device, frequency $5.8 \mathrm{G}$, power consumption $1 \mathrm{~W}$, weight $72 \mathrm{~g}$, with AAT and panel antenna, $15 \mathrm{~km}$ in electromagnetic environment The ultimate transmission distance. The video data in the test is relatively clear. When the drone is flying at a speed of 30 meters and 50 kilometers per hour, it can accurately identify the black body digital sign of $500 \mathrm{~mm} \times 250 \mathrm{~mm}$ on the ground.

\subsection{Airdrop device.}

The airdrop device strives to be simple, reliable and lightweight. The link type latching structure controlled by a digital servo is used in the test. The steering gear is connected to the mechanical connecting rod, and the connecting rod passes through the hook on the load. When the steering gear is commanded, the connecting rod is pulled out from the hook, so that the load can be freely dropped. In the test, the system used $350 \mathrm{~g}$ of pure water as the airborne load to simulate the mission equipment. After several flight tests, the drone can be loaded into a circular area with a radius of $3 \mathrm{~m}$ at a speed of 30 meters and a speed of 50 kilometers per hour, and the hit rate is over $80 \%$.

\subsection{Positioning means.}

The positioning search system consists of four parts: alarm beacon, airborne signal receiver, ground control station and personnel positioning search and rescue device. The drone is equipped with a GPS/Bei Dou navigation signal receiver and a beacon signal receiver, which is connected with the ground control station, and can correct the heading and fly to the beacon in time. At the same time, ground personnel hold high-precision personnel positioning search and rescue devices, relying on drones to correct position information, reducing search and rescue time.

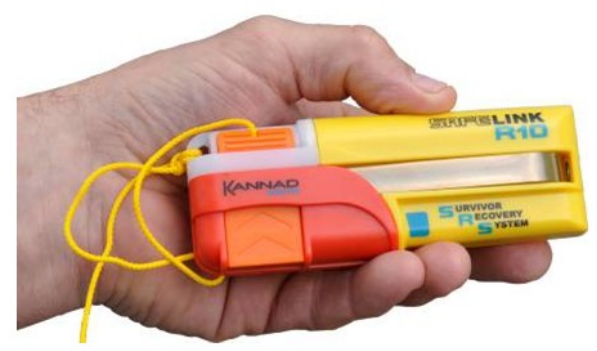

Fig. 2 Type R10 portable AIS emergency indicator 


\section{Conclusion and Outlook}

The collection of rescue work is extremely high. Due to the complexity and change of rescue work, the rescue equipment must also have the characteristics of professionalism and multi-function.

Compared with traditional search and rescue methods, drone search and rescue has the advantages of economy and efficiency.

There is still room for improvement in the UAV positioning search and rescue airdrop system, such as how to ensure signal stability, how to increase the signal transmission distance, etc.

It is believed that with the rapid development of China's economy, the drone market will have a revolutionary development and will penetrate into all aspects of social activities. I believe that more professionals will continue to study in the future.

\section{Acknowledgements}

Fund project: Shenyang Aerospace University Student's Platform for Innovation and Entrepren-eurship Training Program.

First author: Huaqing Liu (1996-- ), male, undergraduate, main research direction is Aeroengine.

Corresponding author: Zhou Yan (1982-- ), male, undergraduate, main research direction is the application of numerical control technology and mechanical design research.

\section{References}

[1] F. Zhang, W. Tong, L.D. Zhou and A.B. Liu: Chinese Medical Equipment: Vol. 31 (2016) No.6, p.175-177. (In Chinese)

[2] J.S. Zhao, W. Mi and S.X. Bai: Chinese Maritime Safety, Vol. 10 (2014), No.8, p.42-44. (In Chinese)

[3] X.D. Chen: China’s Strategic Emerging Industry, Vol. 6 (2018), No.12, p.47. (In Chinese)

[4] C. Zhao: Design and Implementation of Outdoor Autonomous Searching Aircraft (MS., Southwest University of Science and Technology, China 2017), p.8-68. (In Chinese)

[5] T.J. Lei, C.C. Li, and X.Y. He: Journal of Nature Disasters, Vol. 20 (2011) No.1, p.178-183. (In Chinese)

[6] J.H. Zhang, T.X. Wang, T. Xie and S.Y. Fu: Modern Mapping, Vol. 38 (2015) No.6, p.22-24. (In Chinese)

[7] Y.Q. Lai: The Research of UAV Emergency Search System Used in Outdoor(MS., XiJing College, China 2016), p.27-45. (In Chinese)

[8] D.H. Wang, C. Gao and H.C. Zou: Telecommunications technology, Vol. 54 (2014) No.9, p.1204-1209. (In Chinese)

[9] W.T. He: Photelectric Technology Application, Vol. 18 (2003) No.5, p.3-6. (In Chinese)

[10] X.L. Hu: System Engineering Theory and Practice, Vol. 18 (1998) No.6, p.66-69. (In Chinese) 